УДК 532.51

\title{
The 2D Motion of Perfect Fluid with a Free Surface
}

\author{
Victor K. Andreev* \\ Institute of Computational Modelling SB RAS \\ Akademgorodok, 50/44, Krasnoyarsk, 660036 \\ Institute of Mathematics and Computer Science \\ Siberian Federal University \\ Svobodny, 79, Krasnoyarsk, 660041
}

Russia

Received 10.10.2014, received in revised form 10.11.2014, accepted 20.12.2014

The $3 D$ continuous subalgebra is used to searching new partially invariant solution of incompressible perfect fluid equations. It can be interpreted as a non-stationary motion of a plane layer with one free surface. The velocity field and pressure are determined in analytical form by using Lagrangian coordinates.

Keywords: perfect fluid, partially invariant solution, non-stationary motion, free surfaces.

\section{Governing flow equations and main results}

The Euler equations for 2D motions of a perfect fluid are recorded by

$$
\begin{gathered}
u_{t}+u u_{x}+v u_{y}+\frac{1}{\rho} p_{x}=0, \quad u_{x}+v_{y}=0 \\
v_{t}+u v_{x}+v v_{y}+\frac{1}{\rho} p_{y}=0
\end{gathered}
$$

where $\rho$ is the constant fluid density, $u$ and $v$ are the velocity components in the $x$ and $y$ directions, respectively, $p$ is the pressure. The group of point transformations admitted by the system (1) is computed in [1]. Corresponding this group basic continuous Lie algebra includes the three parametrical subalgebra $\left\langle\partial_{x}, t \partial_{u}+\partial_{x}, \partial_{p}\right\rangle$. It has the invariants $t, y, v$ and partly invariant solution of (1) rang two and defect two necessary to seek in the form $u=u(x, y, t)$, $v=v(y, t), p=p(x, y, t)$. From continuity equation $u_{x}+v_{y}=0$ we obtain the relations

$$
u(x, y, t)=u_{1}(y, t) x+u_{2}(y, t), \quad u_{1}(y, t)+v_{y}(y, t)=0 .
$$

Impulse equations (1) are equivalent to the following

$$
\begin{gathered}
u_{1 t}+v u_{1 y}+u_{1}^{2}=f(t), \quad \frac{1}{\rho} p=l(y, t)-f(t) \frac{x^{2}}{2} \\
l_{y}=-v_{t}-v v_{y}, \quad u_{2 t}+v u_{2 y}+u_{1} u_{2}=0
\end{gathered}
$$

with arbitrary function $f(t)$.

Let us introduce the Lagrangian coordinates $(\eta, t)$ by the solving Cauchy problem

$$
\frac{d y}{d t}=v(y, t),\left.\quad y\right|_{t=0}=\eta \text {. }
$$

*andr@icm.krasn.ru

(c) Siberian Federal University. All rights reserved 
We introduce the following denotations

$$
\stackrel{\circ}{u}_{1}(\eta, t)=u_{1}(y(\eta, t), t), \quad \stackrel{o}{u}_{2}(\eta, t)=u_{2}(y(\eta, t), t), \quad \stackrel{o}{v}(\eta, t)=v(y(\eta, t), t),
$$

where $y(\eta, t)$ is a solution of (4). Then the first equations (3) can be reduced to Riccati equation

$$
\stackrel{\circ}{u}_{1 t}+\stackrel{\circ}{u}_{1}^{2}=f(t)
$$

It has general solution

$$
\stackrel{\circ}{u}_{1}(\eta, t)=\frac{\partial}{\partial t}\left\{\ln \left[g(t)\left(1+u_{10}(\eta) \int_{0}^{t} \frac{1}{g^{2}(t)} d t\right)\right]\right\} .
$$

Here $g(t)$ is the solution of the Cauchy problem

$$
g^{\prime \prime}-f(t) g=0, \quad g(0)=1, \quad g^{\prime}(0)=0,
$$

and $u_{10}(\eta)$ is the initial value of function $u_{1}(y, t)$.

The another functions can be found by the formulae

$$
\begin{gathered}
y(\eta, t)=\frac{1}{g(t)} \int_{0}^{\eta}\left[1+u_{10}(\eta) \int_{0}^{t} \frac{1}{g^{2}(t)} d t\right]^{-1} d \eta \\
\stackrel{\circ}{v}(\eta, t)=-\int_{0}^{\eta} \stackrel{o}{u}_{1}(\eta, t) \exp \left[-\int_{0}^{t} \stackrel{o}{u}_{1}(\eta, t) d t\right] d \eta \\
\stackrel{\circ}{u_{2}}(\eta, t)=u_{20}(\eta) \exp \left[-\int_{0}^{t} \stackrel{o}{u}_{1}(\eta, t) d t\right] \\
\stackrel{\circ}{l}(\eta, t)=l_{1}(t)-\int_{0}^{\eta} \stackrel{o}{v}_{t}(\eta, t) \exp \left[-\int_{0}^{t} \stackrel{o}{u}_{1}(\eta, t) d t\right] d \eta
\end{gathered}
$$

with arbitrary function $l_{1}(t)$. So, all unknowns can be determined in analytical form.

Now we show that this solution can be interpreted as an unsteady motion in a strip with one free boundary, see Fig. 1.

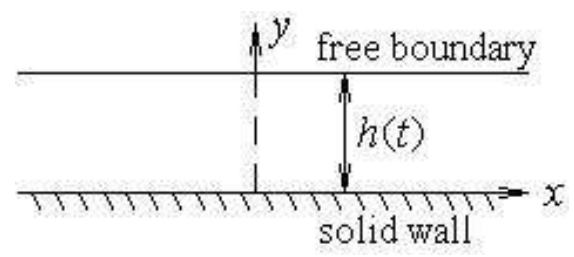

Fig. 1 Geometry of the motion

Really, at the initial time liquid fills the strip of thickness $y=h_{0}=$ const. The line $y=0$ is a rigid wall. Initial velocity field has the form $u_{0}(x, y)=u_{10}(y) x+u_{20}(y), v_{0}(y)=-\int_{0}^{y} u_{10}(y) d y$, $v_{0}(0)=0$. The upper line $y=h_{0}$ is a free boundary and at the initial time the pressure $p\left(h_{0}, 0\right)$ coincides with outer pressure $p_{\text {out }}=p_{10}+p_{00} x^{2} / 2$. For all $t>0$ the strip motion is described 
by the formulae are found above, where $p_{\text {out }}=p_{1}(t)-p_{0}(t) x^{2} / 2$ must be given, so $f(t)=p_{0}(t)$, $f(0)=p_{00}$. The evolution of the free boundary is defined as

$$
h(t)=\frac{1}{g(t)} \int_{0}^{h_{0}}\left[1+u_{10}(\eta) \int_{0}^{t} \frac{1}{g^{2}(t)} d t\right]^{-1} d \eta .
$$

Let us consider two simple cases of the solution (5)-(11), when $u_{10}=a=$ const or $u_{10}=b \eta$, $b=$ const. For the first case the exact solution can be written in Eulerian coordinates as

$$
\begin{gathered}
u(x, y, t)=\frac{\partial}{\partial t} \ln G(t) x+\frac{1}{G(t)} u_{20}(G(t) y), \\
v(y, t)=-\frac{\partial}{\partial t} \ln G(t) y, \\
\frac{1}{\rho} p(x, y, t)=l_{1}(t)+\frac{\partial^{2}}{\partial t^{2}} \ln G(t) \frac{y^{2}}{2}-f(t) \frac{x^{2}}{2},
\end{gathered}
$$

where

$$
G(t)=g(t)\left[1+a \int_{0}^{t} \frac{1}{g^{2}(t)} d t\right]
$$

The equation of the free boundary is

$$
y=h(t)=\frac{h_{0}}{G(t)} .
$$

If we take $g(t)=\cos \omega t, f(t)=-\omega^{2}\left(g(t)=\operatorname{ch} \omega t, f(t)=\omega^{2}\right), \omega=$ const, then the solution exists up to the time $t_{*}=\pi / 2 \omega$ (exists for all time). The solution has to be periodic one if $g(t)=2-\cos \omega t, f(t)=\omega^{2} \cos \omega t(2-\cos \omega t)^{-1}$.

For the second case the formulae have a more complicated shapes and we give here only equation of the free boundary, namely,

$$
y=h(t)=\frac{1}{b g(t) \int_{0}^{t} g^{-2}(t) d t} \ln \left[1+b h_{0} \int_{0}^{t} g^{-2}(t) d t\right] .
$$

Remark 1. In well-known [2] solutions are sought of the shape $\psi(x, y, t)=F(y, t) x+G(y, t)$ for stream function $\left(u_{1}=\psi_{y}, v_{1}=-\psi_{x}\right)$. The unknowns satisfy the eq's

$$
\begin{gathered}
F_{t y}+\left(F_{y}\right)^{2}-F F_{y y}=f_{1}(t), \quad G_{t y}+F_{y} G_{y}-F G_{y y}=f_{2}(t), \\
G=\int U d y-h F+h_{t}^{\prime} y, \quad h_{t t}^{\prime \prime}-f_{1}(t) h=f_{2}(t) .
\end{gathered}
$$

Some particular solution are presented in handbook, see [2, table 13.9, p. 944]. But in this paper we have found exact solution in analytical form.

The problem has a stationary solution. Indeed, the function $v(y)$ satisfies the eq'n $v v_{y y}-v_{y}^{2}=$ $-f_{0}=$ const with general solution
a) $v=\sqrt{\frac{f_{0}}{\left|C_{1}\right|}} \sin \left[\sqrt{\left|C_{1}\right|}\left(C_{2} \pm y\right)\right], \quad f_{0}>0, \quad C_{1}<0$
b) $v= \pm \sqrt{f_{0}}\left(C_{2} \pm y\right)$,
$f_{0}>0, \quad C_{1}=0$
c) $v=\sqrt{\frac{\left|f_{0}\right|}{C_{1}}} \operatorname{ch}\left[\sqrt{C_{1}}\left(C_{2} \pm y\right)\right], \quad f_{0}<0, \quad C_{1}>0$
d) $v=\sqrt{\frac{f_{0}}{C_{1}}} \operatorname{sh}\left[\sqrt{C_{1}}\left(C_{2} \pm y\right)\right], \quad f_{0}>0, \quad C_{1}>0$ 
However, the only case a) has a physical meaning. Really, let us take $h_{0}=\pi / \sqrt{\left|C_{1}\right|}$, then we obtain formulae

$$
\begin{gathered}
u=-\sqrt{f_{0}} x \cos \frac{\pi y}{h_{0}}, \quad v=\frac{h_{0} \sqrt{f_{0}}}{\pi} \sin \frac{\pi y}{h_{0}}, \\
\frac{1}{\rho} p=l_{0}-\frac{f_{0}}{2}\left(x^{2}+\frac{h_{0}^{2}}{\pi^{2}} \sin ^{2} \frac{\pi y}{h_{0}}\right), \quad l_{0}=\text { const }>0,
\end{gathered}
$$

which describe the flow in a strip $0<y<h_{0},|x|<\infty$, with rigid walls $y=0, h_{0}$. The solution obtained is the periodical with respect variable $y$.

\section{Conclusion}

The partially invariant solution of the perfect fluid equations is investigates. This new solution describes the unsteady motion with a free surface. As was shown by examples the solution may has collapse in finite time or to be periodical one. Not that this phenomenon depends on pressure gradient $f(t)$. There has been previous works devoted exact solutions of perfect fluid motions with a free surfaces [3].

This work is supported by the SB RAS (project 44).

\section{References}

[1] A.A.Buchnev, A Lie group admissible for the equations of an ideal incompressible fluid, Dinamika Sploshnoy Sredy, Novosibirsk, (1971), no. 7, 212-214 (in Russian).

[2] A.D.Polyanin, V.F.Zaitsev, Handbook of nonlinear partial differential equations, CRC Press. Taylor and Francis Group, 2012, 942-949.

[3] V.K.Andreev, O.V.Kaptsov, V.V.Pukhnachev, A.A.Rodionov, Application of GroupTheoretical Methods in Hydrodynamics, Kluver Acad. Publ., Dordrecht, Boston, London, 2010.

\section{Двумерное движение идеальной жидкости со свободной поверхностью}

Виктор К. Андреев

\footnotetext{
Непрерывная трёхмерная подалгебра используется для нахождения нового частично инвариантного решения уравнений идеалъной несжимаемой жидкости. Оно интерпретируется как нестационарное движение плоского слоя со свободной поверхностью. При этом поля скоростей и давлений определяются (с помощью переменных Лагранжа) в аналитическом виде.
}

Ключевые слова: идеальная жидкость, частично инвариантное решение, нестационарное движение, свободная поверхность. 